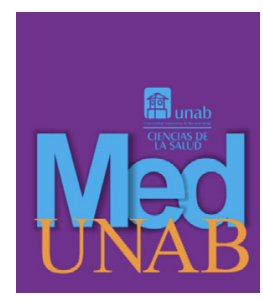

REVISTA DE LA FACULTAD

DE CIENCIAS DE LA SALUD

\title{
Hematoma subgaleal espontáneo en lactantes, una enfermedad subestimada, serie de casos
}

Spontaneous subgaleal hematoma in infants, an underestimated disease, case series

Hematoma subgaleal espontâneo em bebês, uma doença subestimada, série de casos

Lizeth Paola Amaya-Medina, $M D^{1}$ (D), Angélica María Pineda-Parra, $M D^{2}$ (D), Víctor Manuel Mora-Bautista, MD. Esp. ${ }^{3}$ (D)

1. Médica - Cirujana. Bióloga. Urgencias Pediátricas Clínica San Luis. Bucaramanga, Colombia.

2. Médica - Cirujana. Unidad de Cuidado Intensivo Clínica San Luis. Bucaramanga, Colombia.

3. Médico - Cirujano, Especialista en Pediatría. Urgencias pediátricas Clínica San Luis, Docente Cátedra Universidad Industrial de Santander. Bucaramanga, Colombia.

Correspondencia. Víctor Manuel Mora Bautista. Carrera 26 \# 48-56, Clínica San Luis. Teléfono: 3045321812. Email.vmoramd@pediatras.com.co

\section{INFORMACIÓN DEL ARTÍCULO:}

Artículo recibido: 12 de julio de 2020

Artículo aceptado: 26 de mayo de 2021

DOI: https://doi.org/10.29375/01237047.3944

Cómo citar: Amaya-Medina LP, Pineda-Parra AM, Mora-Bautista VM. Hematoma subgaleal espontáneo en lactantes, una enfermedad subestimada, serie de casos. MedUNAB. 2021; 24(2): 255-261 doi: https://doi. org/10.29375/01237047.3944

\section{RESUMEN}

Introducción. Los hematomas subgaleales son patologías poco frecuentes que suelen originarse y resolverse espontáneamente. En neonatos se asocian con distocias y en lactantes o niños mayores con historial de traumatismo, incluso trivial, que puede pasar inadvertido. Hay un pequeño grupo donde no hay antecedente de trauma ni de otros factores como discrasias sanguíneas, se conforma principalmente por lactantes. El objetivo del presente artículo es mostrar una serie de casos de hematomas subgaleales espontáneos en lactantes, por su poca frecuencia, dificultad diagnóstica y diferencias de acuerdo con el tipo neonatal. Casos clínicos. Se presentan cuatro lactantes, entre 4 y 12 meses de edad, diagnosticados con hematomas subgaleales espontáneos (sin relación directa con traumatismo previo), de diferente severidad y atendidos en el servicio de urgencias. Se les realizó una evaluación diagnóstica dirigida a confirmar el hematoma y descartar trastornos de coagulación asociados. Todos los casos mostraron un curso clínico autolimitado sin complicaciones. Discusión. Los hematomas subgaleales espontáneos en lactantes suelen ser benignos, aunque es de vital importancia 
descartar discrasias sanguíneas. Dentro de éstas, los trastornos plaquetarios no suelen tenerse en cuenta y deben estudiarse. Su manejo es conservador y se reabsorberán solos en pocas semanas. Conclusiones. se debe establecer la causa de los hematomas subgaleales en niños pequeños y contemplar su posible aparición espontánea o por causa de discrasias sanguíneas.

Palabras claves:

Hematoma; Lactantes; Cuero Cabelludo; Remisión Espontánea; Informes de Casos.

\begin{abstract}
Introduction. Subgaleal hematomas are rare pathologies that usually arise and resolve spontaneously. In neonates they are associated with dystocia and in infants or older children with a history of trauma, even trivial, which may go unnoticed. There is a small group where there is no background of trauma or other factors such as blood dyscrasias, it is made up mainly of infants. The objective of this article is to show a case series of spontaneous subgaleal hematomas in infants, due to their infrequency, diagnostic difficulty, and differences according to neonatal type. Clinical cases. Four infants are presented, between 4 and 12 months of age, diagnosed with spontaneous subgaleal hematomas (not directly related to previous trauma), of different severity and treated in the emergency unit. They underwent a diagnostic test aimed at confirming the hematoma and ruling out associated coagulation disorders. All cases showed a self-limited clinical course without complications. Discussion. Spontaneous subgaleal hematomas in infants are usually benign, although it is vitally important to rule out blood dyscrasias. Within these, platelet disorders are not usually taken into account and should be studied. Its management is conservative and they will be reabsorbed on their own in a few weeks. Conclusions. The cause of subgaleal hematomas in young children should be established, and their possible spontaneous appearance or due to blood dyscrasias should be considered.
\end{abstract}

Keywords:

Hematoma; Infants; Scalp; Remission, Spontaneous; Case Reports.

\title{
RESUMO
}

Introdução. Os hematomas subgaleais são patologias raras que geralmente surgem e se resolvem espontaneamente. Em recém-nascidos estão associados a distocia e em bebês ou crianças mais velhas com um historial de trauma, mesmo trivial, que pode passar despercebido. Existe um pequeno grupo onde não há historial de traumas ou outros fatores como discrasias sanguíneas, é constituído principalmente por bebês. O objetivo deste artigo é mostrar uma série de casos de hematomas subgaleais espontâneos em bebês, devido à sua infrequência, dificuldade diagnóstica e diferenças de acordo com o tipo neonatal. Casos clínicos. São apresentados quatro bebês, entre 4 e 12 meses de idade, com diagnóstico de hematoma subgaleal espontâneo (não diretamente relacionado a traumas anteriores), de gravidade variável e atendidos no pronto-socorro. Eles foram submetidos a uma avaliação diagnóstica com o objetivo de confirmar o hematoma e descartar distúrbios de coagulação associados. Todos os casos apresentaram evolução clínica autolimitada e sem complicações. Discussão. Os hematomas subgaleais espontâneos em bebês geralmente são benignos, embora seja de vital importância descartar discrasias sanguíneas. Nestas, os distúrbios plaquetários geralmente não são considerados e devem ser estudados. Seu manejo é conservador e serão reabsorvidos por conta própria em algumas semanas. Conclusões. Deve ser estabelecida a causa dos hematomas subgaleais em crianças pequenas e, se considerar seu possível aparecimento espontâneo ou devido a discrasias sanguíneas.

Palavras-chave:

Hematoma; Bebês; Couro Cabeludo; Remissão Espontânea; Relatos de Casos.

\section{Introducción}

Los hematomas subgaleales o colecciones subaponeuróticas fluidas (SFC por su sigla en inglés) en lactantes, son patologías poco frecuentes que surgen por sangrado entre la galea y el laxo tejido conectivo epicraneal. En la mayoría de los casos estas patologías se originan y resuelven espontáneamente, siendo infradiagnosticadas. La mayoría de los reportes hablan de niños menores de 6 meses, con una etiología incierta. No hay un dato preciso de incidencia en lactantes, pero Vaibhav et al. describieron una frecuencia de 2-8 casos por año (1). 
En neonatos el origen de esta patología suele estar relacionado con partos distócicos en los que se monitoriza con electrodos o en los que precisa el empleo de fórceps o ventosa. Estos elementos pueden ser riesgosos para la vida del bebé por coagulopatía y anemización asociadas, aún más si estas se suman a discrasias sanguíneas (donde pueden ocurrir sin historial perinatal) (2). Se han descrito casos tardíos en niños con dichos problemas obstétricos, hasta 3 meses postnatales, la mayoría con curso benigno $(1,3,4)$.

En niños mayores de 6 meses, los casos suelen estar asociados con traumatismos mayores o menores (incluyendo maltrato), malformaciones vasculares o tumores $(1,5)$. La aparición espontánea ( $\sin$ dato de traumatismo), posterior a traumatismos triviales, o tardía con respecto al momento del trauma debe llamar la atención sobre la posibilidad de una discrasia sanguínea (6). No hay datos de la frecuencia con que ocurren estos casos específicos.

Teniendo en cuenta lo anterior, se presentan 4 casos clínicos de lactantes que presentaron hematomas subgaleales espontáneos (HSE), una condición poco frecuente. Esto, con el objetivo de sensibilizar sobre su diagnóstico, sobre las dificultades en cuanto a su etiología, (dado que no se suelen buscar las discrasias sanguíneas) y de sus diferencias respecto del tipo neonatal, a la comunidad médica.

\section{Reportes de caso}

A continuación, se presenta la anamnesis de cada caso. Los datos clínicos, de laboratorio y de seguimiento se detallarán en la Tabla 1.

Tabla 1. Resumen de casos clínicos.

\begin{tabular}{|c|c|c|c|c|}
\hline & CASO 1 & CASO 2 & CASO 3 & CASO 4 \\
\hline Edad & 4 meses & 8 meses & 9 meses & 12 meses \\
\hline Sexo & $\mathbf{F}$ & $\mathbf{F}$ & M & M \\
\hline Localización & TP izquierdo & FP bilateral & P derecho & $\mathrm{F}$ izquierdo \\
\hline Diámetro mayor & $7 \mathrm{~cm}$ & $15 \mathrm{~cm}$ & $7 \mathrm{~cm}$ & $10 \mathrm{~cm}$ \\
\hline Aparición & Súbita & Súbita & Súbita & Súbita \\
\hline Antecedentes & Ninguno & $\begin{array}{c}\text { Trauma leve frontal } \\
2 \text { días atrás }\end{array}$ & $\begin{array}{l}\text { Trauma trivial } \\
4 \text { días atrás }\end{array}$ & $\begin{array}{c}\text { Trauma trivial } 6 \text { días } \\
\text { atrás }\end{array}$ \\
\hline Hemograma & $\begin{array}{c}\text { Leucocitos } 8150 / \\
\text { mm3 } \\
\text { N } 11 \% \text { L } 85 \% \\
\text { Hb } 10.3 \mathrm{~g} / \mathrm{dL} \\
\text { PLT } \\
307,000 / \mathrm{mm} 3\end{array}$ & $\begin{array}{c}\text { Leucocitos } \\
15,340 / \mathrm{mm} 3 \\
\text { N } 27.4 \% \mathrm{~L} 61.7 \% \\
\mathrm{Hb} 12.3 \mathrm{~g} / \mathrm{dL} \\
\text { PLT } \\
321,000 / \mathrm{mm} 3\end{array}$ & $\begin{array}{c}\text { Leucocitos } 8510 / \mathrm{mm} 3 \\
\text { N } 30 \% \text {, L 56\% } \\
\text { Hb } 9.97 \mathrm{~g} / \mathrm{dL} \text { PLT } \\
375,000 / \mathrm{mm} 3\end{array}$ & $\begin{array}{c}\text { Leucocitos } 8860 / \mathrm{mm} 3 \\
\text { N 12\% L } 79 \% \mathrm{Hb} 12.6 \\
\text { g/dL PLT } 282,000 / \\
\text { mm3 }\end{array}$ \\
\hline $\begin{array}{l}\text { Tiempos de } \\
\text { coagulación }\end{array}$ & $\begin{array}{c}\text { PT } 13 \mathrm{~s} \\
\text { INR } 1.3 \\
\text { PTT } 31.2 \mathrm{~s} \\
(\text { control } 35 \mathrm{~s})\end{array}$ & $\begin{array}{c}\text { PT } 12.7 \mathrm{~s} \\
\text { INR } 1.2 \\
\text { PTT } 36.7 \mathrm{~s} \\
(\text { control } 27 \mathrm{~s})\end{array}$ & $\begin{array}{c}\text { PT 14.1 s } \\
\text { INR } 1.11 \\
\text { PTT } 35.2 \mathrm{~s} \text { (control } \\
36 \mathrm{~s})\end{array}$ & $\begin{array}{c}\text { PT } 14.3 \mathrm{~s} \\
\text { INR } 1.04 \\
\text { PTT } 28.8 \mathrm{~s}(\text { Control } \\
32 \mathrm{~s})\end{array}$ \\
\hline $\begin{array}{l}\text { Factores de } \\
\text { coagulación }\end{array}$ & - & $\begin{array}{c}\text { Factor VIII } 63 \%-> \\
74 \%(\mathrm{VR}>50 \%) \\
\text { Factor vW } 77 \%-> \\
67 \%(\mathrm{VR}>42 \%) \\
\text { Cofactor de ristoceti- } \\
\text { na } 75 \%->71 \% \text { (VR } \\
>46.3 \%) \\
(8 \text { meses }->15 \text { meses) }\end{array}$ & - & - \\
\hline Resolución (semanas) & 2 & 4 & 2 & 2 \\
\hline $\begin{array}{l}\text { Seguimiento } \\
\text { hematología }\end{array}$ & No valorado & 6 meses & No valorado & 2 meses \\
\hline
\end{tabular}


Caso 1. Lactante menor femenina de 4 meses que presenta HSE de aparición súbita, de pocas horas de evolución, en región temporoparietal izquierda, sin dolor a la palpación, sin historial de traumatismo, sin antecedentes perinatales ni patológicos de importancia. No hubo otras alteraciones al examen físico. El hemograma y los tiempos de coagulación son normales. Se egresó para seguimiento ambulatorio. El HSE se reabsorbió en 2 semanas.

Caso 2. Lactante menor de 8 meses, sin antecedentes de importancia, quien dos días atrás sufrió una caída de la cama y desarrolló un hematoma en región frontal, en la línea media, el cual había resuelto. Consulta por la aparición de gran HSE que compromete el área del hematoma inicial, se extiende a las regiones frontal y biparietal rodeando la fontanela anterior y abarcando hasta la región supraciliar(Figura 1). El resto del examen físico fue normal. Se obtuvieron exámenes con reportes normales y una tomografía corroboró el hematoma subgaleal. Dado el tamaño de la lesión la menor fue remitida a hematología para valoración con exámenes complementarios de resultados normales. El hematoma desapareció en cuatro semanas. Finalmente, la paciente fue dada de alta por hematología 6 meses después tras nuevo seguimiento con exámenes normales.

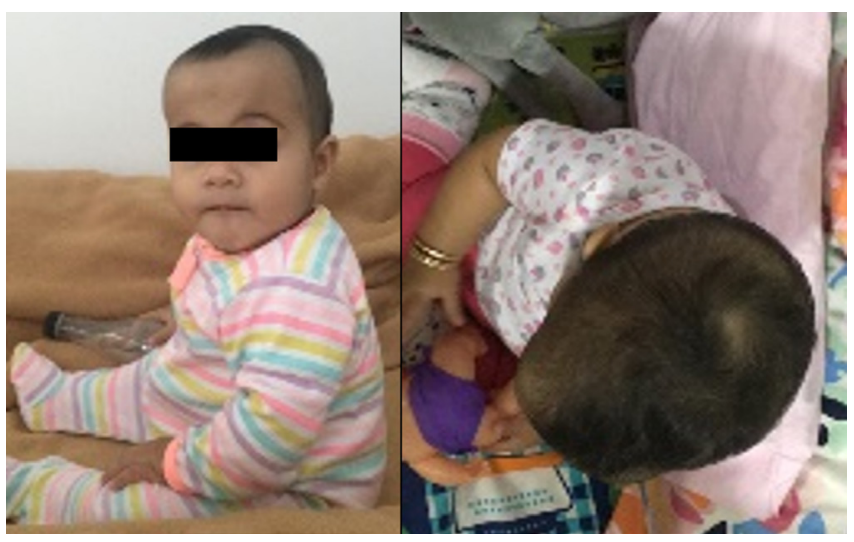

Figura 1. Hematoma subgaleal del caso 2.

Fuente: elaboración propia.

Caso 3. Lactante menor, masculino, de 9 meses, sin historial médico relevante, quien sufrió traumatismo leve al deslizarse de la cuna hace 4 días. Ingresa a la institución por presentar por cinco horas edema en región parietal derecha, de aparición espontánea. No hubo otros hallazgos en el examen físico. Como diagnóstico se consideró HSE y se solicitaron exámenes que arrojaron resultados normales. Se obtuvo ecografía de tejidos blandos que mostró lesión compatible con el diagnóstico. El hematoma resolvió tras dos semanas.
Caso 4. Lactante mayor, sin antecedentes médicos, masculino, de 12 meses, con aparición espontánea de hematoma subgaleal en región frontal izquierda (Figura 2). Presentó un trauma cráneo encefálico seis días atrás que no dejó lesiones. Sin ningún otro síntoma asociado. La evaluación física no arroja alteraciones. Se realizaron exámenes que arrojaron reportes normales. El paciente fue valorado por hemato-oncología dado el tamaño de la lesión pero, pasada una semana, la lesión se aplana por lo que no le solicitaron exámenes adicionales. La lesión desapareció a las dos semanas de evolución y el paciente fue dado de alta por dicha especialidad dos meses después.

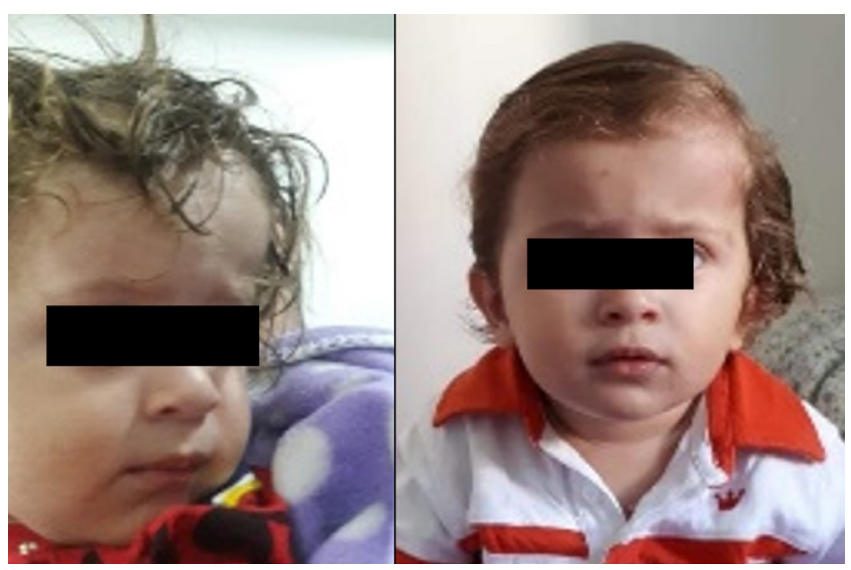

Figura 2. Hematoma subgaleal del caso 4

Fuente: elaboración propia.

\section{Discusión}

El hematoma subgaleal en lactantes es una patología infrecuente que puede ser subdiagnosticada y subvalorada por su curso benigno y rápida resolución. Este tipo de lesión se localiza en la profundidad del tejido subcutáneo del cráneo y aumenta su densidad de forma circunferencial y ligeramente asimétrica. $\mathrm{Su}$ distribución es difusa. La lesión atraviesa las suturas craneales y tiene la forma de una luna creciente, lo cual se aprecia bien en las tomografías (7).

En los casos de HSE en lactantes, es decir sin trauma directo ni discrasias sanguíneas, se presume que se origina por ruptura de venas emisarias por distorsión o tracción. Estas son lesiones microscópicas que, por la laxitud de la gálea y una vasculatura relativamente más abundante en estos niños, facilitan el sangrado espontáneo o posterior a un traumatismo ocurrido varios días atrás. Este último caso es una asociación discutible y se propone como hipótesis (5).

Para propósitos clínicos se considera que el HSE es espontáneo si el trauma no desencadenó el hematoma 
inmediatamente ni en las primeras 24 horas postrauma. Con relación a este punto, en tres de los cuatro casos se documentó antecedente de traumatismo trivial con más de 48 horas de anticipación, lo cual desestima su relación causal. Cabe resaltar que en estos casos algunos autores conjeturan que la causa directa podría ser un traumatismo menor inadvertido como peinar o halar el cabello, donde también se rompen vénulas (8-11).

Los reportes existentes de hematomas subgaleales se centran en los lactantes con historial obstétrico, donde la mayoría de los pacientes no supera los 3 meses de edad y se presume la existencia de microfracturas con sangrado y fístulas microscópicas de LCR. Los casos en lactantes mayores, escolares y adolescentes usualmente involucraron traumatismos directos, evidentes en su mayoría y espontáneos sólo en un $7.5 \%$ de los reportes ( 7 de 106 casos, Tabla 2). Se reportaron peores desenlaces cuando se intervinieron (el hematoma mismo se comporta como un tapón hemostásico y drenarlo facilitó la recurrencia), recuperaciones espontáneas entre dos y cuatro semanas, algunas asociaciones con trombopatías transitorias y un ligero predominio en el sexo femenino (Tabla 2) $(1,4,9,11,12)$.

Tabla 2. Casos reportados de hematoma subgaleal pediátrico no asociado a discrasias sanguíneas

\begin{tabular}{|c|c|c|c|c|c|c|}
\hline Autor & Casos & Edades & Sexo & Ubicación* & Asociación & Resolución \\
\hline Kuban (1983) & 6 & $8 m-8 a$ & $\begin{array}{l}3 \mathrm{M} / \\
3 \mathrm{~F}\end{array}$ & F, P, OT, FTPO & $\begin{array}{l}\text { a. Caída } \\
\text { b. Golpe contra borde } \\
\text { c. Golpe contra la } \\
\text { pared } \\
\text { d. Presión contra una } \\
\text { ventana } \\
\text { e. Espontáneo }\end{array}$ & $2-3$ semanas \\
\hline $\begin{array}{l}\text { Kirkpatrick } \\
\text { (1986) }\end{array}$ & $\begin{array}{l}\text { Hamlin (1968): } 1 \\
\text { Cantu (1971): } 1 \\
\text { Adeloye (1975): } 50 \\
\text { Faber (1976): } 1 \\
\text { Falvo (1981): } 2 \\
\text { Beauchamp (1983): } \\
1 \\
\text { Okafor (1984): } 1 \\
\text { Reporte actual: } 1\end{array}$ & $\begin{array}{l}3 \mathrm{a} \\
15 \mathrm{a} \\
2 \mathrm{~m}-15 \mathrm{a} \\
6 \mathrm{a} \\
4 \mathrm{y} 9 \mathrm{a} \\
4 \mathrm{a} \\
17 \mathrm{a} \\
12 \mathrm{a}\end{array}$ & $\begin{array}{l}\mathrm{F} \\
\mathrm{M} \\
\mathrm{F}\end{array}$ & $\begin{array}{l}\text { FTP derecho } \\
\text { F izquierdo } \\
\text { F, P, O, T } \\
\text { F derecho } \\
\text { FTPO bilateral } \\
\text { FTP anterior bilat } \\
\text { FTPO bilateral } \\
\text { FT bilateral } \\
\text { FTPO bilateral }\end{array}$ & $\begin{array}{l}\text { Maltrato } \\
\text { Halar el cabello } \\
\text { Contusión craneal } \\
\text { Halar el cabello } \\
\text { Espontáneo en ambos } \\
\text { casos } \\
\text { Peinado } \\
\text { Patada de karate } \\
\text { Trombopatía }\end{array}$ & $\begin{array}{l}\mid \\
\mid \\
\mid \\
\mid \\
2 \text { semanas }\end{array}$ \\
\hline Cooling (1991) & 1 & $23 \mathrm{~m}$ & M & FTPO bilateral & Contusión craneal & 2 semanas \\
\hline Jimeno (2007) & 1 & $9 \mathrm{~m}$ & M & P izquierdo (abscedado) & Espontáneo & $\begin{array}{l}2 \text { semanas } \\
\text { (ameritó cirugía) }\end{array}$ \\
\hline Koizumi (2010) & 1 & $15 \mathrm{a}$ & M & FTP derecho & Espontáneo & $\begin{array}{l}5 \text { semanas } \\
\text { (embolización, } \\
\text { aspiración) }\end{array}$ \\
\hline Vaibhav (2010) & $\begin{array}{l}\text { Hopkins (2002): } 6 \\
\text { Schoberer (2008): } 5 \\
\text { Reporte actual: } 4\end{array}$ & $\begin{array}{l}1-4,5 \mathrm{~m} \\
7 \mathrm{a} \\
1-2 \mathrm{~m} \\
1-3 \mathrm{~m}\end{array}$ & $\begin{array}{c}\mathrm{M} \\
\mathrm{F} \\
2 \mathrm{M} / \\
3 \mathrm{~F} \\
3 \mathrm{M} / \\
1 \mathrm{~F}\end{array}$ & $\begin{array}{l}\text { FTPO bilateral } \\
\text { P izquierdo } \\
\text { FTPO bilateral } \\
\text { FTPO y P bilat. }\end{array}$ & $\begin{array}{l}\text { Trauma obstétrico } \\
\text { Trauma en ceja izq } \\
\text { Trauma obstétrico } \\
\text { Trauma obstétrico }\end{array}$ & $\begin{array}{l}\mid 2-4 \text { semanas } \\
4-20 \text { semanas } \\
3-8 \text { semanas }\end{array}$ \\
\hline Wang (2016) & 9 & $1-11 \mathrm{~m}$ & $\begin{array}{l}4 \mathrm{M} / \\
5 \mathrm{~F}\end{array}$ & $\mathrm{O}, \mathrm{F}, \mathrm{FP}, \mathrm{F}$ bilateral & $\begin{array}{l}\text { Trauma obstétrico } \\
\text { Uno (1) espontáneo }\end{array}$ & $2-20$ semanas \\
\hline $\operatorname{Er}(2017)$ & 1 & $8 \mathrm{a}$ & $\mathrm{F}$ & FTPO bilateral & Halar el cabello & 3 semanas \\
\hline Ferreira (2017) & 1 & $2 \mathrm{~m}$ & $\mathrm{~F}$ & $\mathrm{O}$ & Espontáneo & 8 semanas \\
\hline Haidar (2017) & 1 & $7 \mathrm{a}$ & $\mathrm{F}$ & FP bilateral & Espontáneo & 4 semanas \\
\hline
\end{tabular}




\begin{tabular}{|c|c|c|c|c|c|c|}
\hline \multirow{10}{*}{ Scheier (2019) } & JAMA (1934): 1 & $8 \mathrm{a}$ & M & & & $>52$ semanas \\
\hline & Scott (1936): 1 & $\mathrm{NR}^{* *}$ & NR & & & NR \\
\hline & Hamlin (1941): 1 & $6 \mathrm{a}$ & $\mathrm{F}$ & & & NR \\
\hline & Madhu (1990): 1 & $13 \mathrm{a}$ & M & Masivos, no precisa & & 1 semana \\
\hline & Yip (2003): 1 & $13 \mathrm{a}$ & M & ubicaciones. Varios con & Todos: Halar el & 2 días (cirugía) \\
\hline & Vu (2004): 1 & $8 \mathrm{a}$ & $\mathrm{F}$ & proptosis y alteraciones & cabello & 6 semanas \\
\hline & Onyeama (2009): 1 & $31 \mathrm{~m}$ & $\mathrm{~F}$ & movilidad ocular & & 2 semanas \\
\hline & Edmonson (2016): 1 & $16 \mathrm{a}$ & M & & & 5 d (cirugía) \\
\hline & Pui (2019): 1 & $13 \mathrm{a}$ & M & & & 1 semana \\
\hline & Scheier (2019): 1 & $10 \mathrm{a}$ & $\mathrm{F}$ & & & 2 semanas \\
\hline $\begin{array}{l}\text { De Vito } \\
(2020)\end{array}$ & 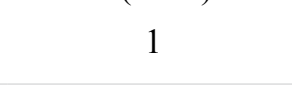 & $11 \mathrm{a}$ & $\mathrm{F}$ & F bilateral & $\begin{array}{c}\text { Halar el cabello } \\
\text { (trenzas) }\end{array}$ & NR \\
\hline $\begin{array}{l}\text { Patchana } \\
(2020)\end{array}$ & 1 & $12 \mathrm{a}$ & $\mathrm{F}$ & $\mathrm{F}$ bilateral & $\begin{array}{c}\text { Halar el cabello } \\
\text { (trenzas) }\end{array}$ & $\begin{array}{c}2 \text { semanas (ci- } \\
\text { rugía) }\end{array}$ \\
\hline
\end{tabular}

* F: frontal, T: temporal, P: parietal, O: occipital. ** NR: No referido

Fuente: elaboración propia.

El diagnóstico diferencial se establece con caput sucedaneum, cefalohematomas, angioedema, celulitis y neoplasias $(12,13)$. Se debe recordar, si hubo traumatismo craneal, que podría ser el reflejo de una fractura incluso cuando se presente tardíamente (14). La anamnesis, el examen físico y el uso de ecografía, tomografía computarizada o resonancia magnética, en los casos donde haya duda diagnóstica, constituyen el abordaje recomendado $(1,12)$. En los dos casos en que se realizaron imágenes diagnósticas los hallazgos fueron compatibles con hematoma y no hubo evidencia de fracturas. Por el contrario, se recomienda no realizar estudios de imagen ni de laboratorio en los casos de hematoma subgaleal donde la causa y el diagnóstico son claros (1).

En los casos de HSE es recomendable descartar trastornos de coagulación $(1,3,4)$. En los niños de los casos abordados en este texto los tiempos de coagulación fueron normales por lo que se descartan estas enfermedades. La coagulopatía más común es una deficiencia en el factor de Von Willebrand aunque puede ser también el debut de una hemofilia, especialmente en neonatos (15), o de alteraciones de factores menos comunes como el factor V o el factor XIII $(16,17)$. También se ha descrito en anemia de células falciformes, secundario a infarto de hueso craneal $(18,19)$.

Como dato relevante, se menciona que en HSE sin coagulopatía se han descrito trombopatías subyacentes, transitorias o permanentes (6). Dada la evolución benigna de los casos presentados, se podría considerar adecuada la decisión de no evaluar trombopatías ni medición de otros factores de coagulación. Por otro lado, habría sido conveniente que todos los casos fueran evaluados por hematología, lo cual constituye una limitación del estudio en dos de los casos revisados. En los HSE el manejo siempre es conservador y se sabe que incluso cuando se trata de discrasias sanguíneas esta se reabsorberá por completo en unas semanas $(1,13)$. Sólo se deberían intervenir si hay dolor severo, necrosis del colgajo, descompensación hemodinámica o signos de infección $(6,8)$. Se ha descrito el uso de terapia endovascular para casos refractarios (20).

\section{Conclusiones}

Los hematomas subgaleales en lactantes pueden ser espontáneos o asociados a traumatismos triviales e inadvertidos. En estos casos resulta vital descartar discrasias sanguíneas, principalmente hemofilias. Para descartarlas se recomienda realizar tiempos de coagulación y valoración en consulta de hematología. Si no es claro que la lesión corresponda a hematoma se pueden hacer imágenes diagnósticas. En los casos donde la causa es clara (trauma directo o historial obstétrico/perinatal) no se deben realizar estudios imagenológicos ni de laboratorio. En general, el manejo es conservador y los hematomas se reabsorberán solos en pocas semanas.

\section{Responsabilidades éticas}

Protección de personas $\mathbf{y}$ animales: el presente estudio se considera una investigación sin riesgo dada su naturaleza. Fue aprobado por el Comité de Ética Institucional.

Confidencialidad de los datos: los autores declaran que han seguido los protocolos de su centro de trabajo sobre la publicación de datos de pacientes.

Derecho a la privacidad y consentimiento informado: los autores han obtenido el consentimiento informado 
de los pacientes y/o sujetos referidos en el artículo. Este documento obra en poder del autor de correspondencia referidos en el artículo.

\section{Conflicto de intereses}

Los autores declaran no tener conflicto de intereses.

\section{Referencias}

1. Vaibhav A, Smith R, Millman G, Cooper J, Dwyer J. Subaponeurotic or subgaleal fluid collections in infancy: an unusual but distinct cause of scalp swelling in infancy. BMJ Case Rep [Internet]. 2010 Nov 2;2010:bcr0420102915. https://doi.org/10.1136/ bcr.04.2010.2915

2. Reid J. Neonatal Subgaleal Hemorrhage. Neonatal Netw. 2007;26(4):219-27. https://doi. org/10.1891/0730-0832.26.4.219

3. Smith A, Kandamany N, Okafor I, Robinson I, Foran A, McNamara R. Delayed Infant Subaponeurotic (Subgaleal) Fluid Collections: A Case Series of 11 Infants. J Emerg Med [Internet]. 2016;50(6):881-6. https://doi.org/10.1016/j.jemermed.2016.03.024

4. Wang S, Drake J, Kulkarni A V. Management and outcome of spontaneous subaponeurotic fluid collections in infants: the Hospital for sick children experience and review of the literature. J Neurosurg Pediatr. 2016;18(October):442-7. https://doi. org/10.3171/2016.4.PEDS1641

5. Patchana T, Ghanchi H, Taka T, Calayag M. Subgaleal hematoma evacuation in a pediatric patient: A case report and review of the literature. Surg Neurol Int. 2020;11(243):1-5. https://doi.org/10.25259/ SNI $207 \quad 2020$

6. Esteller M, López N, Chiandetti A, Martínez-Roig A. Hematoma subgaleal espontáneo como manifestación de una alteración de la función plaquetaria. An Pediatr. 2014;80(1):4-5. https://doi.org/10.1016/j. anpedi.2011.10.020

7. Lacoma Latre EM, Sánchez Lalana E, Alconchel Lagranja AP. Hematoma subgaleal difuso. Imagen Diagn. 2016;7(2):72-3. https://doi.org/10.1016/j. imadi.2016.02.003

8. Er A, Çağlar A, Akgül F, Ulusoy E, Karslı E, Yılmaz $D$, et al. A Rare Cause of Subgaleal Hematoma in Children: Hair Pulling. Turkish J Pediatr Emerg Intensive Care Med. 2017;4(1):33-5. https://doi. org/10.4274/cayd.54254

9. Scheier E, Ben-Ami T, Guri A, Balla U. Subgaleal Hematoma from a Carnival Costume. Isr Med Assoc J. 2019;21(6):422-3. Disponible en: http://www.ncbi. nlm.nih.gov/pubmed/31280516
10. De Vito A, Mankad K. Our experience of subgaleal haematoma due to hair pulling. Acta Paediatr Int J Paediatr. 2020;109(2):426. https://doi.org/10.1111/ apa. 15033

11. Scheier E, Guri A, Balla U. Subgaleal haematoma due to hair pulling: Review of the literature. Acta Paediatr Int J Paediatr. 2019;108(12):2170-4. https://doi. org/10.1111/apa.14971

12. Jimeno A, García-Lechuz Moya JM, González E. Lesión cefálica de partes blandas en un niño de 9 meses. Enferm Infecc Microbiol Clin. 2008;26(3):175-6. https://doi.org/10.1157/13116755

13. Ferreira AM, Silva J, Monteiro V, Tavares S, Araújo R, Rocha C. Cranial Swelling - A diagnostic Challenge (Portugués). Nascer e Crescer - Birth Growth Med J. 2017;26(2):53-6. https://doi.org/10.25753/ BirthGrowthMJ.v26.i2.9942

14. Chida K, Yukawa H, Mase T, Endo H, Ogasawara $\mathrm{K}$. Spontaneous slow drainage of epidural hematoma into the subgaleal space through a skull fracture in an infant. Neurol Med Chir (Tokyo). 2011;51(12):854-6. https://doi.org/10.2176/nmc.51.854

15. Altuna D. Hemostasia neonatal: manifestaciones clínicas y causas del sangrado neonatal. Hematología [Internet]. 2017;21(E):101-9. Disponible en: http:// www.sah.org.ar/revista/numeros/vol21/extra3/16vol21-extra noviembre.pdf

16. Kim D, Taragin B. Subgaleal hematoma presenting as a manifestation of Factor XIII deficiency. Pediatr Radiol. 2009;39(6):622-4. https://doi.org/10.1007/ s00247-009-1176-y

17. Panigrahi S, Mishra SS, Das S, Patra SK. Large subgalealhematomaaspresentation of parahemophilia. J Neurosci Rural Pract [Internet]. 2013;4(2):240-2. http://doi.org/10.4103/0976-3147.112785

18. Mishra SS, Senapati SB, Gouda AK, Behera SK, Patnaik A. Spontaneous extradural and subgaleal hematoma: A rare neurosurgical crisis of sickle cell disease. Asian J Neurosurg [Internet]. 2017;12(1):47. https://doi.org/10.4103/1793-5482.144177

19. Fortin-Boudreault R-P, Story E. Spontaneous Subgaleal Hematoma: An Unusual Complication of Sickle Cell Disease. J Clin Case Reports [Internet]. 2016;06(01):1-2. $\quad$ https://doi.org/10.4172/21657920.1000681

20. Koizumi H, Suzuki S, Utsuki S, Nakahara K, Niki J, Mabuchi I, et al. A case of non-traumatic subgaleal hematoma effectively treated with endovascular surgery. Interv Neuroradiol. 2010;16(3):317-21. https://doi.org/10.1177/159101991001600315 